\title{
Uncertainty span for relative permeability and capillary pressure by varying wettability and spatial flow directions utilizing pore scale modelling
}

\author{
Thomas Ramstad, Anders Kristoffersen, and Einar Ebeltoft
}

Equinor ASA, Norway

\begin{abstract}
Relative permeability and capillary pressure are key properties within special core analysis and provide crucial information for full field simulation models. These properties are traditionally obtained by multi-phase flow experiments, however pore scale modelling has during the last decade shown to add significant information as well as being less time-consuming to obtain.

Pore scale modelling has been performed by using the lattice-Boltzmann method directly on the digital rock models obtained by high resolution micro-CT images on end-trims available when plugs are prepared for traditional SCAL-experiments. These digital rock models map the pore-structure and are used for direct simulations of two-phase flow to relative permeability curves.

Various types of wettability conditions are introduced by a wettability map that opens for local variations of wettability on the pore space at the pore level. Focus have been to distribute realistic wettabilities representative for the Norwegian Continental Shelf which is experiencing weakly-wetting conditions and no strong preference neither to water nor oil. Spanning a realistic wettability-map and enabling flow in three directions, a large amount of relative permeability curves is obtained. The resulting relative permeabilities hence estimate the uncertainty of the obtained flow properties on a spatial but specific pore structure with varying, but realistic wettabilities.

The obtained relative permeability curves are compared with results obtained by traditional SCALanalysis on similar core material from the Norwegian Continental Shelf. The results are also compared with the SCAL-model provided for full field simulations for the same field. The results from the pore scale simulations are within the uncertainty span of the SCAL models, mimic the traditional SCAL-experiments and shows that pore scale modelling can provide a time- and cost-effective tool to provide SCAL-models with uncertainties.
\end{abstract}

\section{Introduction}

Wettability is a key factor for multiphase flow behaviour in porous media in general, and for reservoir rocks especially. In the origin of the petroleum industry, clastic reservoir rocks where assumed to behave waterwet due to the water-wetting minerals composing the rock and pore surface. Since the 70'ties, several papers [see i.e $1,2]$ have questioned this assumption by adsorption of oil components on the pore surface due to braking the thin water-film established between the oil-phase and the rock surface [3]. It is now well acknowledged that the wettability of clastic reservoir rocks experiences weakly wetting behaviour and generally no strong preference to neither oil nor water.

Wettability can be determined and studied by different approaches [2-8]. The most common for the petroleum industry have been either measurements of contact angles, $\theta$, and interfacial tension, $\sigma / \mathrm{IFT}$, [4] or macroscopic experiments and measurements on core samples by the traditionally Amott/Amott-Harvey and USBM methods $[9,10,11]$. The benefit of the first approach is that the wetting preference is well defined, however the major and crucial - drawback is that the measurements must be done on a flat surface with a geometry far from the geometry and shape of the pore structure in porous media. Macroscopic measurements are done on core samples exposed to real fluids at reservoir conditions, however the measurements are not regarded as well-suited to define the specific wettability where limited preferences to neither of the phases are experienced - which is the case for most clastic reservoir rocks. Even though the macroscopic approach has its limitation, it is the most used method by the industry due to its pragmatic approach and it provides reliable quality information of the wettability preference.

A huge amount of wettability definitions has been suggested during the years: mixed-wet, intermediate-wet, neutral-wet, weakly water-wet, weakly to strongly waterwet etc. Even though the origin of these definitions was intended to be well-defined, the pragmatic use of the terms is not. When providing SCAL-models for full-field applications, the methods and pragmatic understanding described in ref. [2-8] are generally applied. The core sample is generally cleaned, irreducible water saturation, $\mathrm{S}_{\mathrm{wir}}$, is established, the core sample is then saturated with reservoir oil at reservoir conditions - and subsequently aged for two to three weeks at irreducible water saturation. The core is then, by definition,

\footnotetext{
Corresponding author: trams@equinor.com
} 
restored to its original wetting conditions. This approach is well established, even though the distribution of the (irreducible) water-film is not known and the local wettability in the pore space, pore throats, body and pore necks are not known.

SCAL data for use in reservoir modelling are commonly obtained through core flooding experiments. Detailed information about wettability is distributed in the core sample, and how that influence the fluid distributions inside the pore space, is limited. Recent advances in Xray $\mathrm{CT}$ technology have made non-destructive imaging of core material readily available in the petroleum industry. High resolution micro-computed tomography (micro-CT) enables both resolving the pore space to a high degree of accuracy and visualize the distribution of fluids at the pore level - before and after a flooding. Such technology provides a valuable tool for interpreting and augmenting SCAL data. Micro-CT images can in addition be segmented into pore scale models that can be used for flow simulations. To optimally utilize the results from this new imaging equipment, a digital workflow that combines experimental imaging with simulations directly on these images will be essential.

In this paper we combine the approach of using the well-defined wettabilities by contact angles and interfacial tension distributed on the porous structure of the rock surface obtained by micro-CT imaging and direct pore scale simulations.

Wettability maps are generated, where wettabilities can be varied for different parts of the pore structure and/or varied from sample to sample, experiencing identical pore structure. This will provide additional and important information how the various definitions of wettabilities influence the dynamic flow behaviour, i.e. relative permeability and capillary pressure.

\section{Methods}

In this section we present the background and techniques for the different steps in the workflow. Every step provides useful information, but ultimately, we wish to gain direct information about essential SCAL data like relative permeability and capillary pressure for different wettability and orientations.

The total workflow contains the following steps:

- Sample selection and preparation

- High resolution Micro-CT imaging

- Image segmentation

- Direct pore scale flow simulations in the segmented images

\subsection{Samples and preparation}

End trims from representative core material were selected as starting point for the workflow. The core material has been part of a SCAL program and comes from two different wells in the same formation on the Norwegian Continental Self (NCS).

The end-trims themselves are incomplete and otherwise not suited for flooding experiments. However, they can be imaged in micro-CT and thus provide pore scale images used for further modelling.

Examples of overview scans and sample selection area are shown in Fig. 1. Coarse micro-CT were first done to evaluate the integrity of the material and find suited parts for sub-sampling. The samples were embedded in epoxy solution before further handling to avoid alterations in the pore space of loosely consolidated grains. After epoxy treatment, micro-plugs with diameter $5 \mathrm{~mm}$ were drilled for further imaging. Presently, no confining pressure can be applied which may affect the pore throats and measured permeability.

The plug preparation and scanning were carried out by a FEI HeliScan ${ }^{\circledR}$ micro-CT instrument.

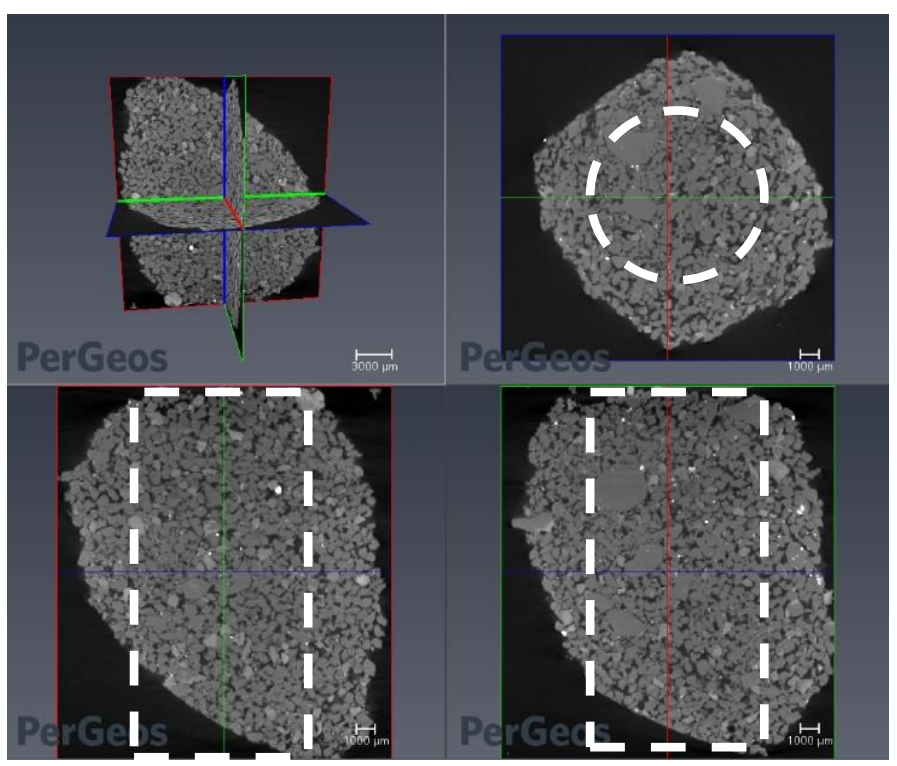

Fig. 1 Overview micro-CT scan of an end-trim sample. The micro-plug selection is marked.

\subsection{MCT, segmentation and downsampling}

The $5 \mathrm{~mm}$ samples were scanned at a photon energy of $100 \mathrm{kV}$ and the resolution was $4.2 \mu \mathrm{m} / \mathrm{pixel}$. 


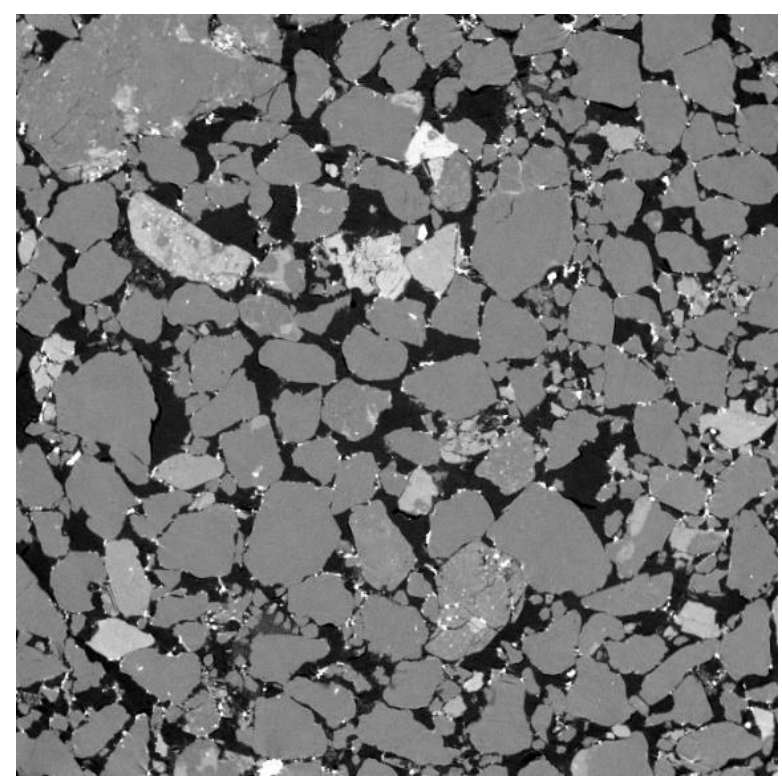

Fig. 2 High resolution micro-CT scan of an end-trim sample.

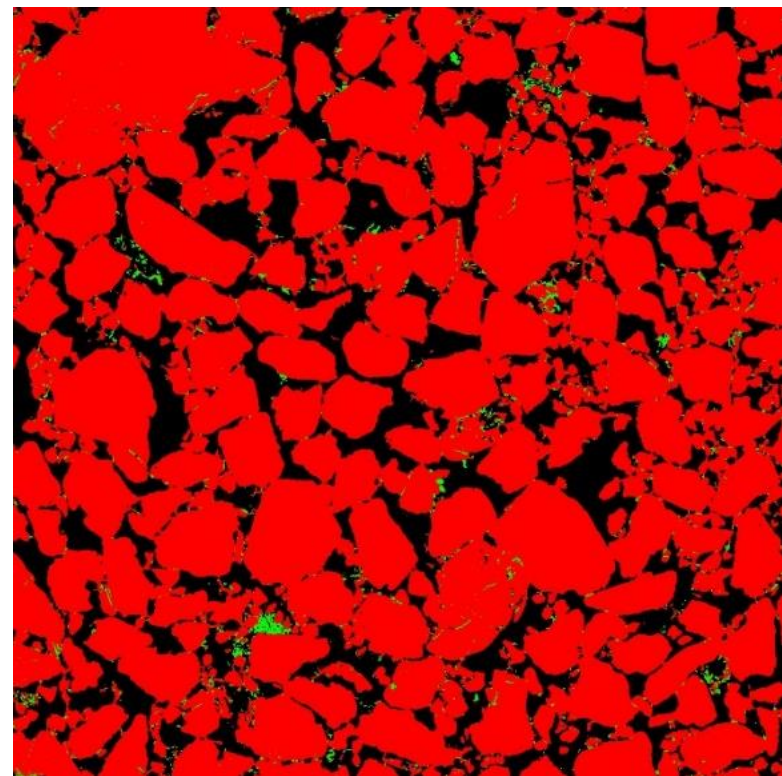

Fig. 3 The image in Fig. 2 segmented into a solid phase (red), pore phase (black) and a sub-resolution porosity phase, which mainly corresponds to clay (green).

The segmentation was performed in the Avizo ${ }^{\circledR}$ software, using a marker-controlled watershed algorithm. An example of a raw image and the corresponding label image is shown in Fig. 2 and Fig. 3, respectively. The image is segmented into three phases (solid, pore and subresolution porosity). The lattice-Boltzmann algorithm inputs a binary map (solid/pore). Therefore, the subresolution porosity was assigned to the solid phase. The reason is that the sub-resolution porosity is clay, and we do not expect clay-bound water to contribute to the flow. It should also be noted that the clay content was small in all of the examined samples as can be seen in Fig. 3.

Lattice Boltzmann modelling is computationally intensive, and it is crucial to limit the size of the grid. Therefore, it was decided to downsample the images prior to the flow simulations. By downsampling to a coarser resolution, it is possible to model a larger physical section of the plug, thus coming closer to a representative elementary volume.

Fig. 4 shows a slice at the original resolution and one that is downsampled. Visually, there seems to be no significant loss of information. To verify this, the relative permeabilities were calculated on the same physical sample at three different resolutions; $7 \mu \mathrm{m}, 10 \mu \mathrm{m}$ and 15 $\mu \mathrm{m}$ (data not shown). Indeed, the results were equal. It was therefore concluded that the downsampling approach was justified. The length dimensions of the samples are listed in Table 1.

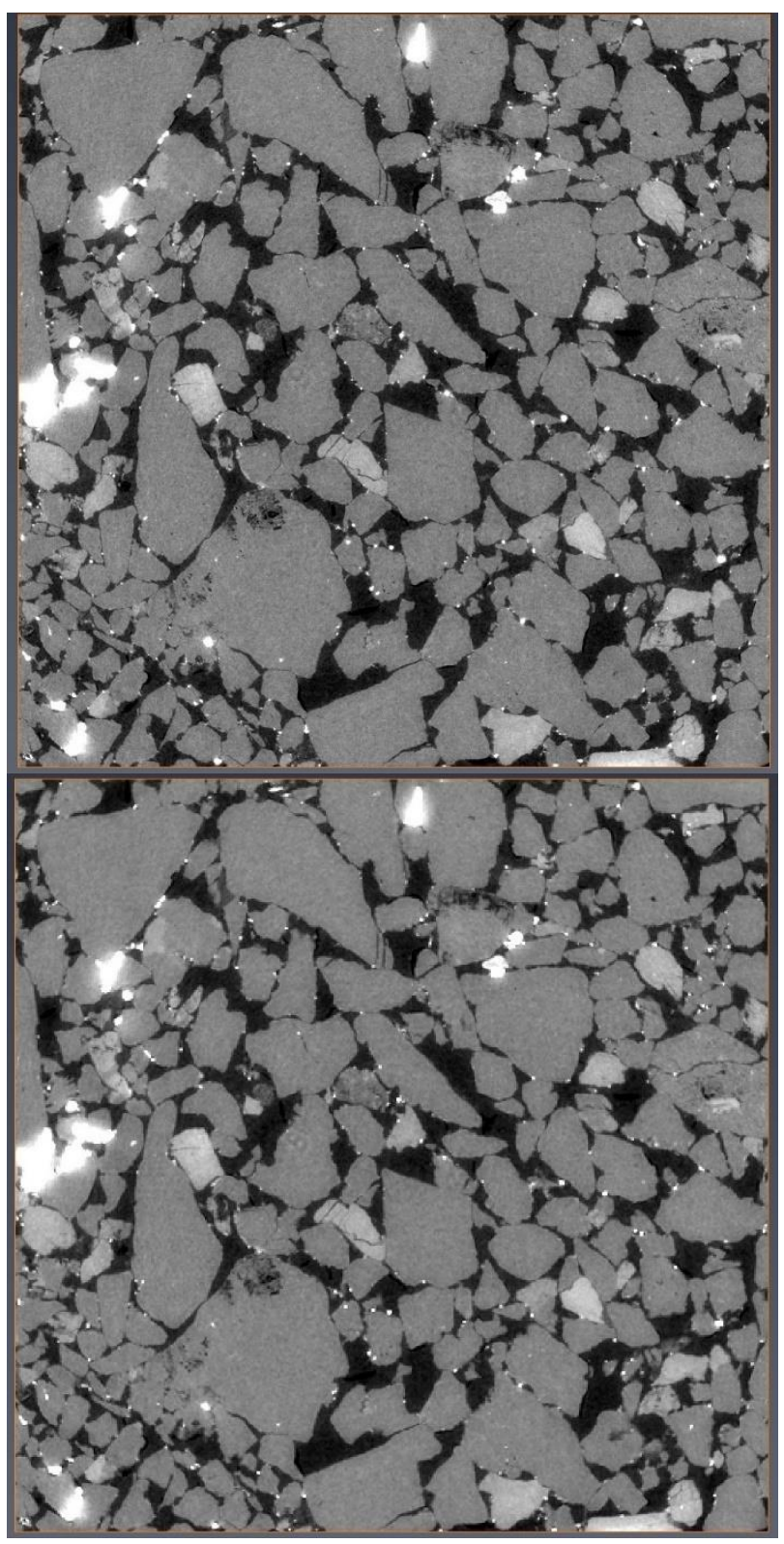

Fig. 4. An image slice at the original resolution of 4.2 $\mu \mathrm{m}$ (top) and the corresponding slice downsampled to a resolution of $15 \mu \mathrm{m}$ (bottom). 


\subsection{Direct pore scale simulations}

Multiphase flow is simulated directly on the segmented images from the micro-CT scans. We use a multiphase lattice Boltzmann method (LBSIM) for this purpose, and the simulations perform the basis for the SCAL results produced.

The lattice Boltzmann method is a kinetic approach to fluid dynamics and is well documented in the literature [16]. The method is well suited for simulating multiphase flow in complex geometries and fluid interfaces that are the cases for flow in porous media. From such simulations it is possible to obtain capillary pressure and relative permeability that can be compared to different experimental set-ups.

For the multiphase simulations presented in this paper we use the following set-up:

1. Irreducible water saturation is established through primary drainage simulations

2. Wettability in the model is established either as a uniform wettability or through a local wettability distribution on the pore space, i.e. wettability map

3. Water flooding capillary pressure curves are obtained through forced imbibition

4. Relative permeability points are generated from a steady-state set-up. The results are then analysed using the software Sendra ${ }^{\circledR}$ [12] with the end-point obtained from the capillary pressure simulations.

The different steps of the workflow can be repeated with different conditions, and the simulations thus provide a fast tool for sensitivity testing, such as wettability and spatial flow direction.

The forced imbibition capillary pressure simulations are done by injecting water with a fixed pressure drop over the model. When the water saturation has converged, the capillary pressure is recorded, and the applied pressure drop increased. This way we obtain the capillary pressure curve and the end-point residual saturations.

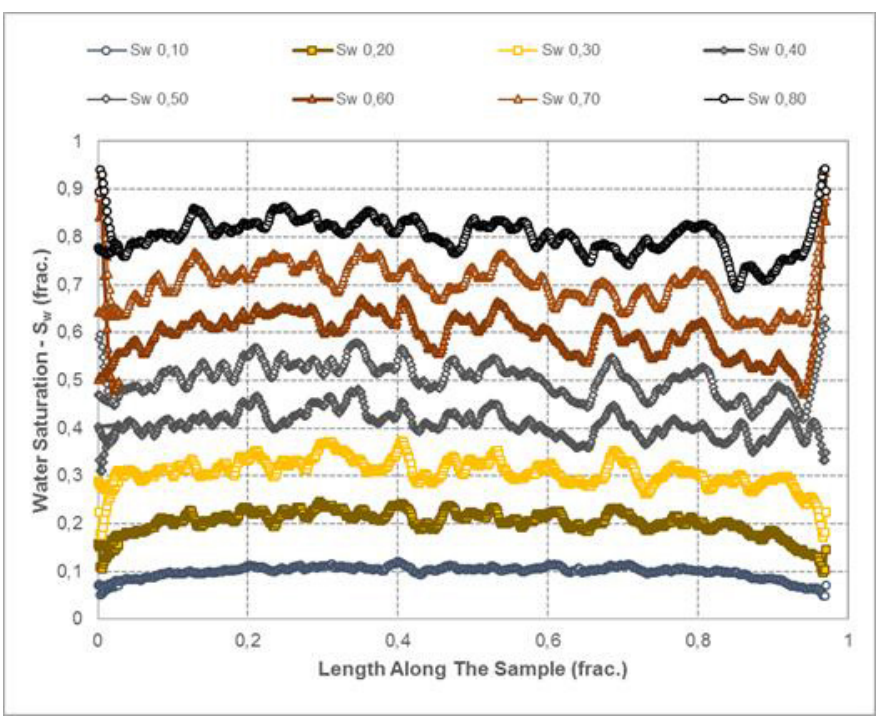

Fig. 5. Water saturation profile at steady state in the simulations.

The steady state relative permeability curves are done with periodic boundary conditions to minimize endeffects. When the effective permeability of each phase has converged, the saturation is changed to preserve the fluid distribution history. This is done to model the proper effect of steady state water flooding with increasing water saturation.

As shown in Fig. 5, the saturation distribution in the sample is close to uniform, and end-effects are relatively small despite the limited physical size of the sample.

Steady state effective permeability values are normalized with the initial oil effective permeability at irreducible water saturations to get relative permeability. The steady state set-up is suited for obtaining the relative permeability in the mid saturation region. However, it suffers from resolution limitations closer to low end-point values. To compensate for this, a curve fit is done combining steady state points with residual points from the capillary pressure simulations.

\subsection{Wettability distribution}

One of the main aims of this study is to investigate the effects of wettability and to clarify some of the concepts related to wettability distribution. With the use of rock images and modelling, we can set the wettability locally on the grains and investigate the resulting fluid distributions.

In LBSIM the wettability is defined through a scalar affinity value that controls the local wettability. The local affinity values range from 1.0 to -1.0. On a plain surface, this correlation can be translated into a contact angle between the fluid and solid pore walls. However, for complex and rugged grain surface it is difficult to define 
unique contact angle. Hence, it is common to refer a local wettability of 1.0 to strongly water wet (WW) while a value of -1.0 relates to strongly oil wet $(\mathrm{OW})$. In terms of global wettability for the sample, there are two ways of setting that in the simulations, which we refer to as: Uniform wettability and non-uniform wettability.

For a uniform wettability the local wetting parameter is constant throughout the sample. This is indeed a special case, as there are many local effects that can alter wettability, like e.g. fluid composition and mineralogy. However, a uniform wettability can be justified in cases of a clear wettability trend in the entire sample like strongly WW or strongly OW.

A more sophisticated way of handling the wettability is by distribution of the local wettability parameters in the pore space. Such a control of the local wettability opens for a more in-depth treatment of wettability effects in a setting of non-uniform wettability.

There are many ways to define the wettability in the samples, but in this study, the local wettability parameters are limited to a static binary wettability map as shown in Fig. 6.

The wettability maps consist of two affinity parameters: OW and WW, and they only apply to the fluid grid voxels that border to solid. The OW and WW are distributed according to the fluid distribution at initial water saturation, $\mathrm{S}_{\mathrm{wi}}$. Grid voxels where the oil is in contact with the grain is set to OW while the WW is applied elsewhere.

We chose to set the WW parameter to $\mathrm{WW}=1.0$ while the OW will vary from 1.0 to -1.0 . This to mimic an aging process. Changing wettability though an aging is a slow process that usually takes weeks. Hence, the values of OW and WW are kept static and do not change throughout a simulation.

A resulting fluid distribution is shown in Fig. 7. We can observe the curvature of droplets and the contact with grains. This is further magnified in Fig. 9 and Fig. 10. Such fluid distributions can again be compared directly to micro-CT images of flooding experiments (see e.g. ref [19]).

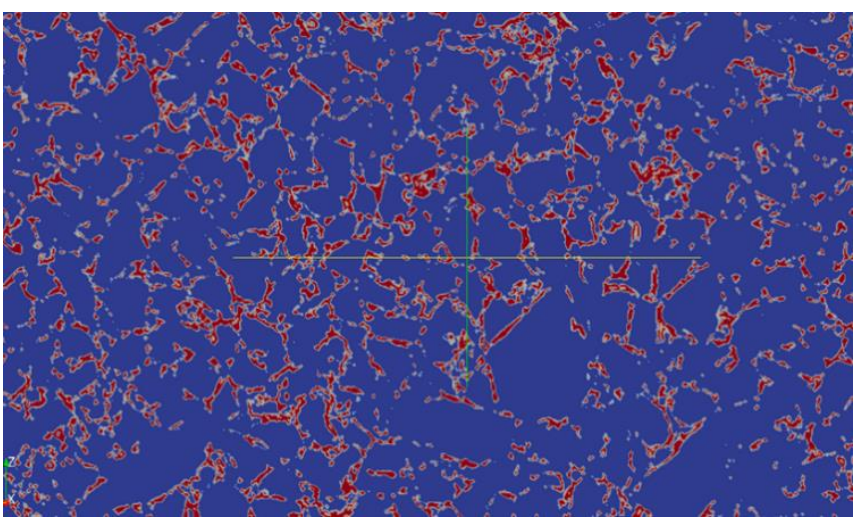

Fig. 6 Binary wettability map where red denotes OW index and blue is WW index. Only voxels that border to solid grains will be affected by the wettability index.

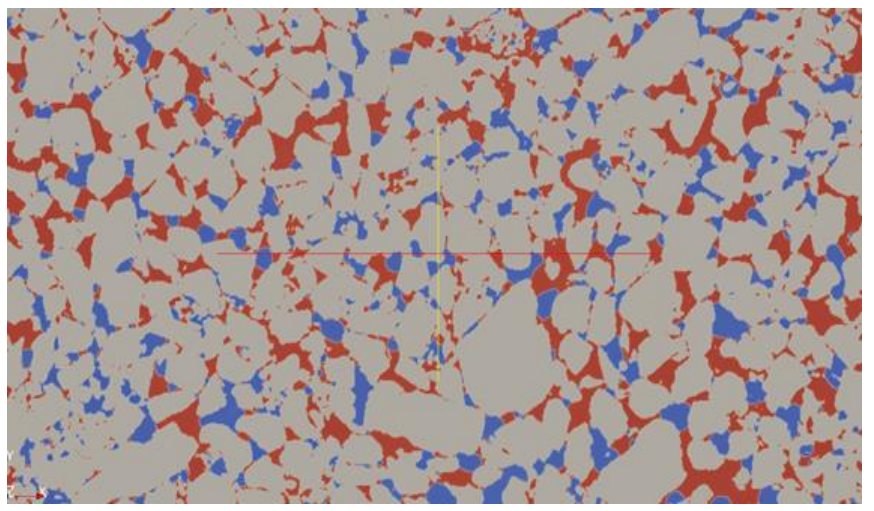

Fig. 7. A resulting fluid distribution from the wettability map in Fig. 6. The water saturation is $S_{w}=0.5$. Water is blue and oil is red.

\section{Results}

This section provides the results obtained by combining Micro-CT images, spatially pore scale simulations combined with wettability distribution within the porous structure.

\subsection{Models and Set-up}

We have used the workflow and tools listed in Section 2 to build pore scale models and run two-phase direct flow simulations with LBSIM. The dimensions and properties of the models are listed in Table 1:

Table 1. Basic properties of the digital models

\begin{tabular}{|c|c|c|c|}
\hline Sample & A & B & C \\
\hline $\begin{array}{c}\text { Size } \\
\text { [voxels] }\end{array}$ & $\begin{array}{c}485 \times 485 \\
\times 1085\end{array}$ & $\begin{array}{c}485 \times 485 \\
\times 878\end{array}$ & $\begin{array}{c}485 \times 485 \times \\
878\end{array}$ \\
\hline $\begin{array}{c}\text { Resolution } \\
{[\text { microns }]}\end{array}$ & 15 & 15 & 15 \\
\hline
\end{tabular}


Dynamic SCAL-experiments utilizing either the steady state- or the unsteady state techniques are generally exposed to capillary end-effects and are not well suited to provide estimates of the residual oil saturation, $\mathrm{S}_{\text {orw }}$. A common approach is to combine such flow experiments with comparable centrifuge or porous plate techniques which are well suited to provide estimates of residual saturations [13]. This behaviour is also experienced for steady state pore scale modelling on digital images. Hence, it tends to be unsuited for determination of very low relative permeabilities close to residual saturations, $\mathrm{S}_{\text {wir }}$ and $\mathrm{S}_{\mathrm{orw}}$, both because of low fractional flow and limited resolution. The steady state dynamic simulations are here done at typical capillary numbers of $\mathrm{Ca}=10^{-5}$.

Pore network analogues can reduce some of the problems related to limited resolutions, but these come at the cost of simplifying the pore scale micro structure and reducing dynamic effects for low capillary numbers [18].

Similar approach as for traditional SCAL-experiments have been utilized in the simulations. Capillary pressure with the coherent saturation end-points are determined prior to determination of the relative permeabilities. These latter simulations are performed with steady state conditions and anchored in the saturation end-points, $S_{\text {orw }}$, determined from the capillary pressure simulations.

When the pore scale models are built, it is straight forward to change input parameters related to wettability and sample orientation and re-run simulations from the same starting point, i.e. the configuration after primary drainage. With the data points obtained from capillary pressure and relative permeability simulations, we have used the software Sendra ${ }^{\circledR}$ for further curve fitting.

The simulations have been done on end-trims from core material defining the SCAL-model used for full-field simulations. The obtained curved are then compared to relevant field SCAL model with uncertainty bands [20].

From the samples listed in Table 1 with sensitivity on wettability and orientation for each sample, we obtain a total of 54 relative permeability curves and 9 capillary pressure curves. These are used to in-depth analyse the impact by wettability on SCAL data and the associated uncertainty in interpretation of SCAL data.

\subsection{Capillary pressure and end-points}

All capillary pressure data points from the simulations are obtained through a forced imbibition set up. The pressure drop over the model is fixed, and when the time dependent saturation profile has converged, both the resulting saturation and pressure are recorded.

Fig. 8 shows the capillary pressure points determined by pore scale modelling and subsequently curve fitted by the
LET-correlation for capillary pressure [14]. This provide guidelines for where to anchor residual oil saturation, $\mathrm{S}_{\text {orw }}$, for the relative permeabilities shown in the next section. In addition, the capillary pressure curves are also determined.

The forced imbibition simulations were done with uniform wettability distribution in the intermediate wet range. From the curves, there is a relatively long range of water saturations with negative Pc values.

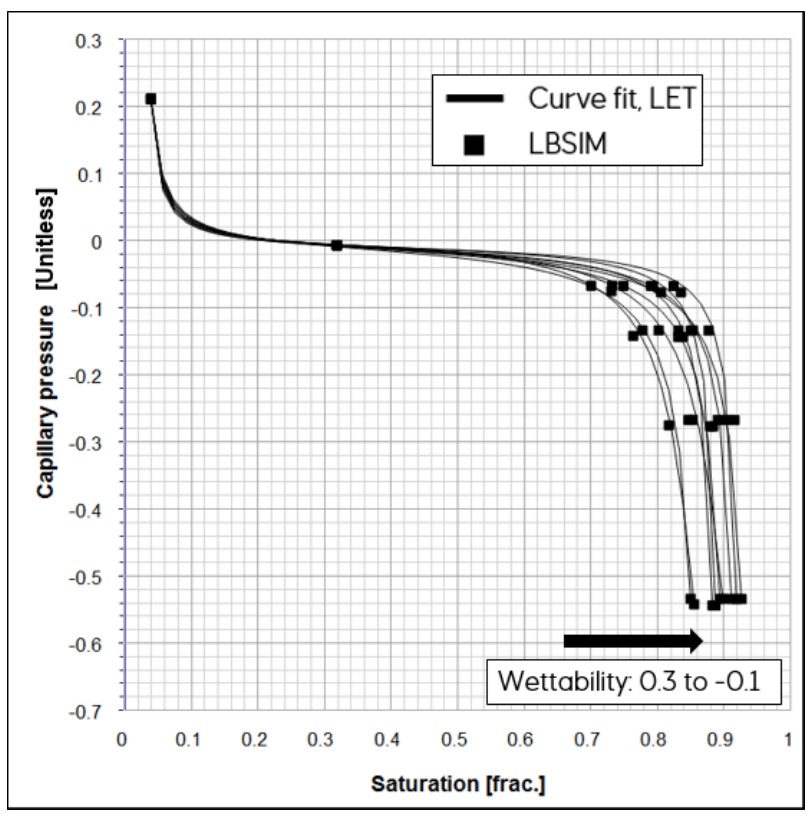

Fig. 8 Capillary pressure curves for all samples with different wettabilities on the intermediate wet range. The end-points are listed in Table 2.

The residual oil saturation, $\mathrm{S}_{\text {orw }}$, experiences reliable values for $\mathrm{S}_{\text {orw }} \epsilon[0.06-0.15]$ and are listed in Table 2 . These end-points are used in further interpretation of steady state relative permeability curves.

Table 2. End-point saturations from the capillary pressure curves.

\begin{tabular}{|c|c|c|c|}
\hline Sample & A & B & C \\
\hline Swir $_{\text {wir }}$ & 0.04 & 0.04 & 0.04 \\
\hline Sorw $_{\text {OW }}=-0.5$ & 0,088 & 0,105 & 0,149 \\
\hline Sorw $_{\text {oW }}=0.3$ & 0,080 & 0,073 & 0,101 \\
\hline Sorw, $_{\text {oW }=0.5}$ & 0,112 & 0,116 & 0,144 \\
\hline
\end{tabular}

\subsection{Relative permeabilities}

Relative permeabilities are obtained by pore scale simulations (LBSIM) with steady state flow conditions. 
Each of these samples utilized different wettabilities, and simulations have been done for three spatial directions

A total of six different wettabilities have been used, three cases of uniform wettability and three cases of nonuniform wettability where a binary wettability maps, as described in section 2.4, have been applied. For the uniform wettability cases, the local wettability constant takes the values $0.3,0.1$ and -0.2 giving intermediate global wettabilities. These correspond to the capillary pressure curves shown in Fig. 8 .

In the cases of the non-uniform wettability the parameter WW (see Section 2.4) has been defined to strictly 1.0 and the OW parameter takes the values $0.5,0.3$ and -0.5 . The wettability maps are generated from the $S_{\text {wir }}$ distributions, and hence the WW only applies to small pores and corners in pore bodies.

With the OW index taking the value -0.5 , the resulting fluid distribution can be observed in detail Fig. 10. A wetting configuration that is more towards a global oil wet condition is observed, and water droplets forming on the grain walls are observed.

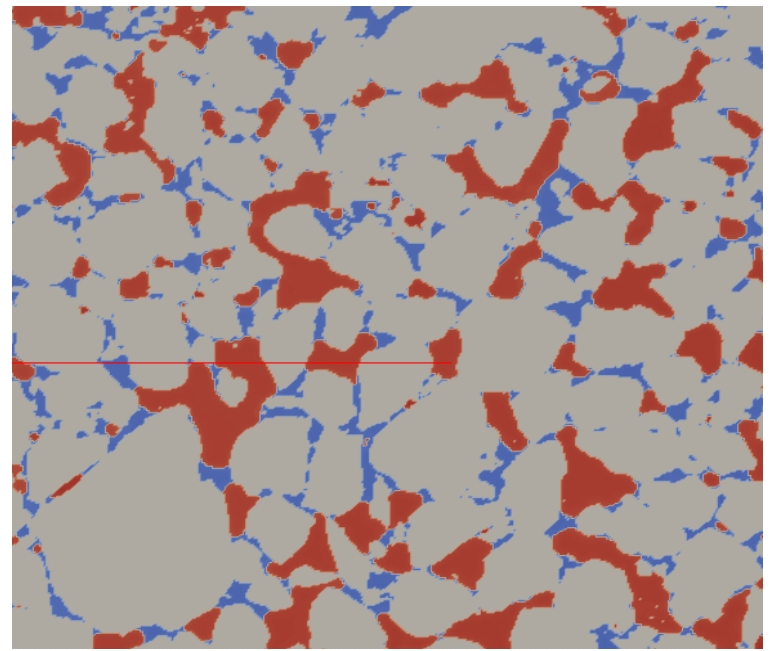

Fig. 9 Different fluid distributions from non-uniform wettability $\mathrm{OW}=0.5$

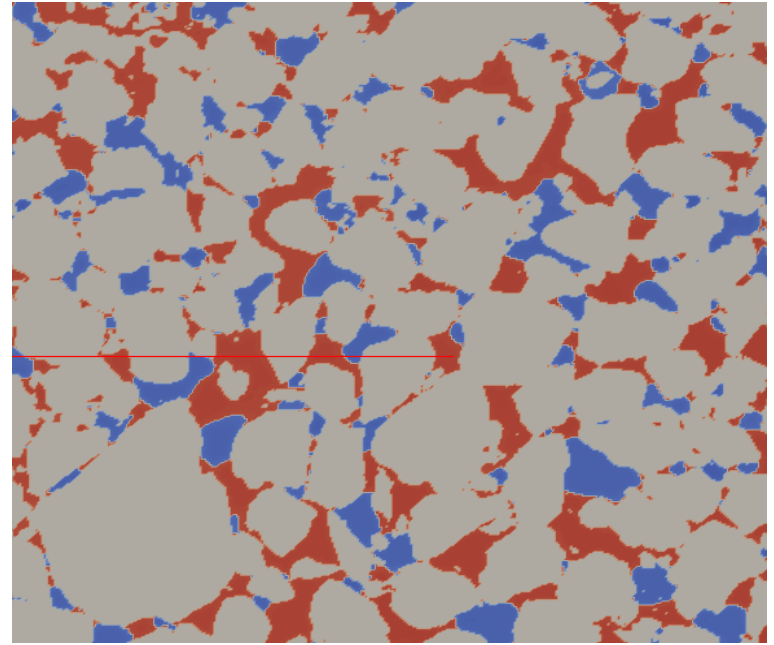

Fig. 10 Different fluid distributions from non-uniform wettability $\mathrm{OW}=-0.5$
With $\mathrm{OW}=0.5$, the oil resides on the pore bodies as droplets formed by the water wet films and corners, but there is not a large curvature for the interfaces between water and oil in large pores (Fig. 9).

These two wettability settings have been compared to the SCAL models in Fig. 11 and Fig. 12. All relative permeabilities obtained by LBSIM have been curve fitted by the LET-correlation for relative permeability [15]. The observation is that the uniform wettability case produces more symmetric relative permeability curves and the relative permeability of water $\left(\mathrm{K}_{\mathrm{rw}}\right)$ is consistently higher than the SCAL model close to residual oil saturation, see Fig. 11. For the non-uniform wet cases the bundle for curves stay within the uncertainty of the SCAL model, $\mathrm{K}_{\mathrm{rw}}\left(\mathrm{S}_{\mathrm{wir}}\right)$ is lower than for the uniform wettability case, albeit on the pessimistic side of the SCAL model, see Fig. 12.

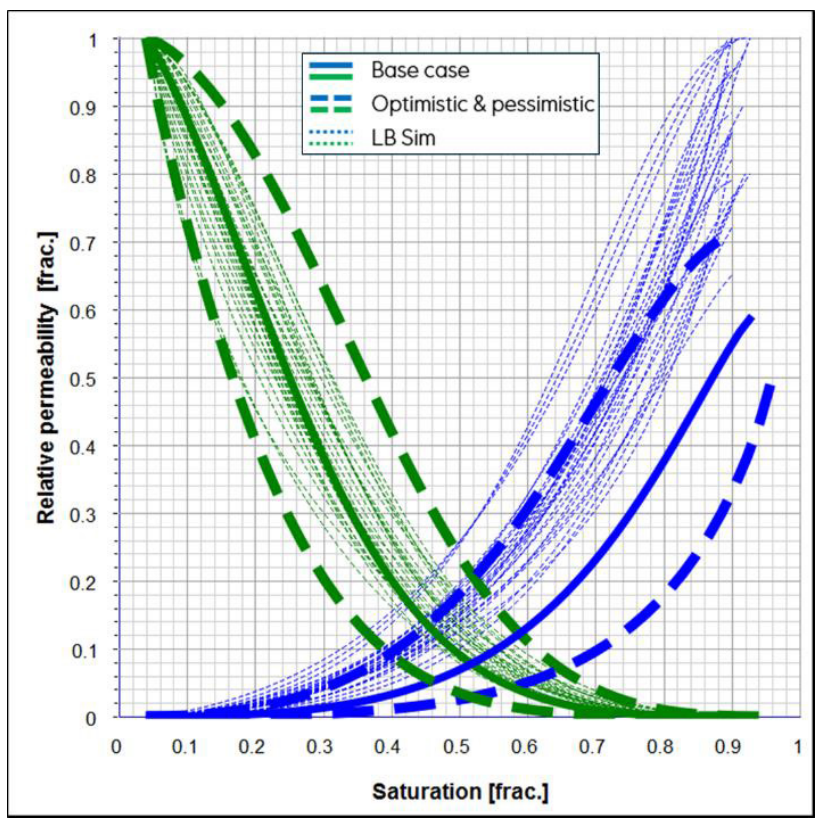

Fig. 11 All relative permeability for curves for the uniform wet cases. 


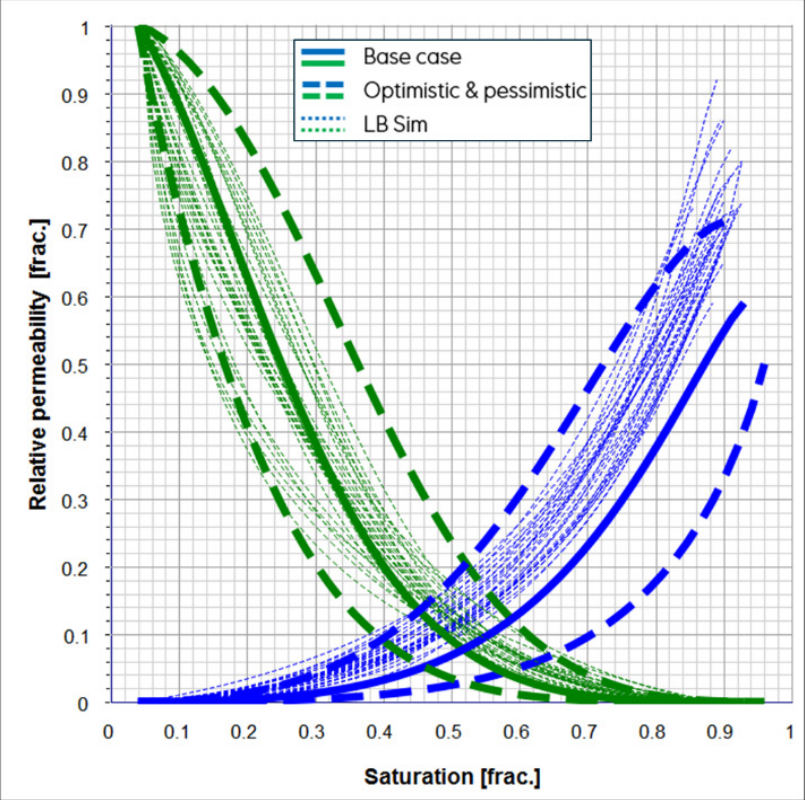

Fig. 12 All relative permeability for curves for the nonuniform wet cases.

In the following we will focus on the sensitivity to wettability using the approach of wettability maps with non-uniform wettability. All results from pore scale modelling non-uniform wet conditions are within the provided uncertainty span for the SCAL-model, even though it can be argued that the water relative permeabilities and oil relative permeabilities are on the pessimistic and optimistic side, respectively. This observation is similar to the results for the comparison to steady state experiments from one individual core - Fig. 13 and Fig. 14 - and is considered for further work when defining the wettabilitiy maps.

As for the individual interpretation the results are within the expected uncertainty for SCAL-experiments and multi-phase flow behaviour in general. The steady state experiments were performed with the following fraction flow of water, $f_{w}=0.008 ; 0.06 ; 0.22 ; 0.5 ; 0.78 ; 0.94$; $0.992 ; 1$.

The relative permeablity curves tend to be overestimated for low values - toward the residuals - compared to the experimental results. This is due to limited resolution and finite-size effects in the models and fluid dynamics.

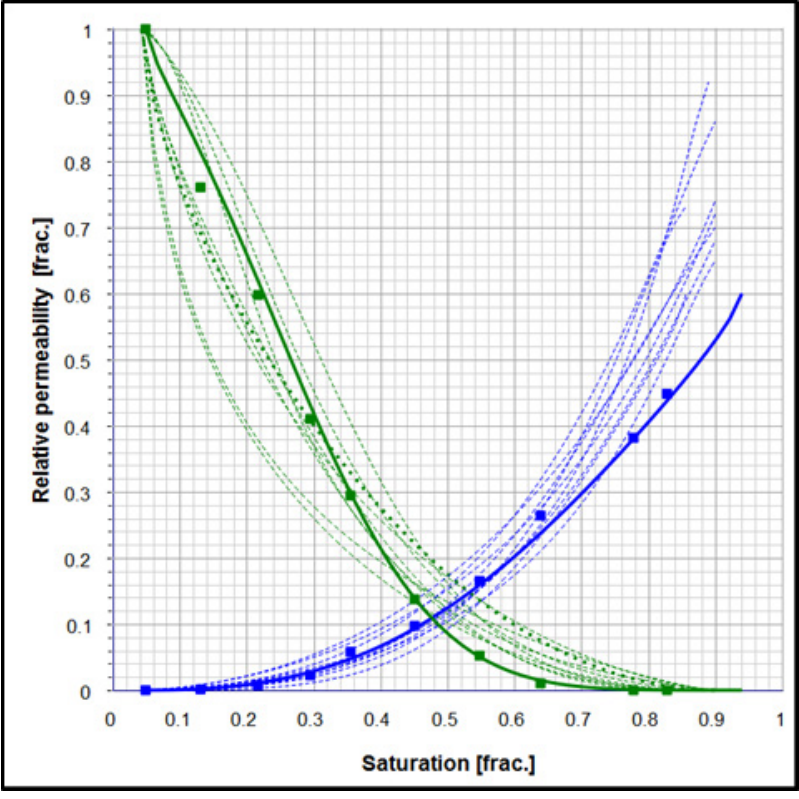

Fig. 13 Experimental steady state relative permeability curves compared to different non-uniform wet conditions and orientations, lin-lin.

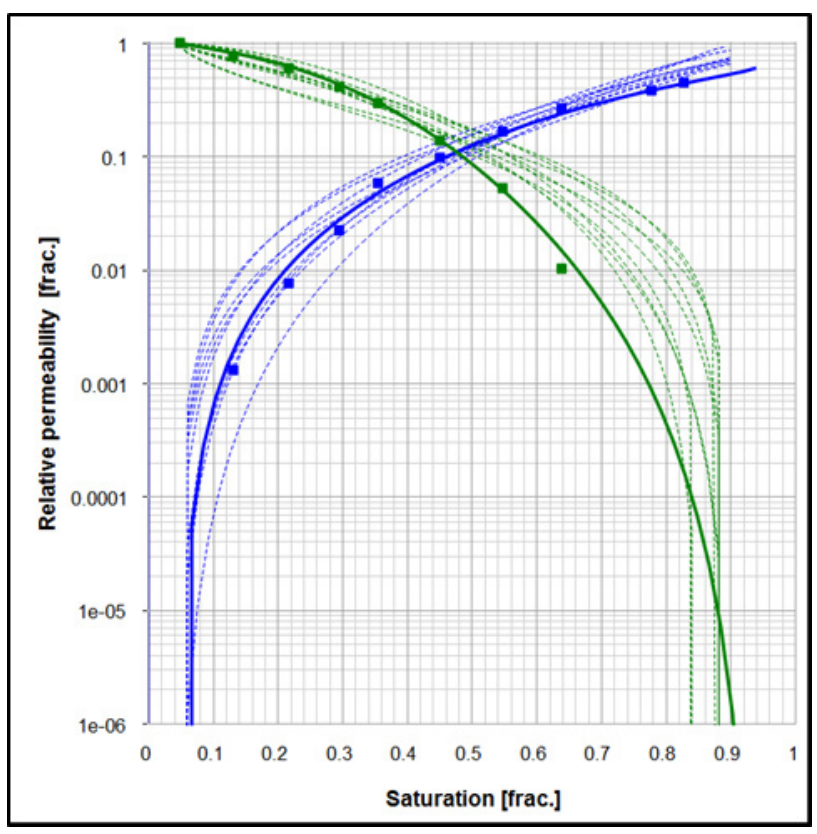

Fig. 14 Experimental steady state relative permeability curves compared to different non-uniform wet conditions and orientations, semilog. 


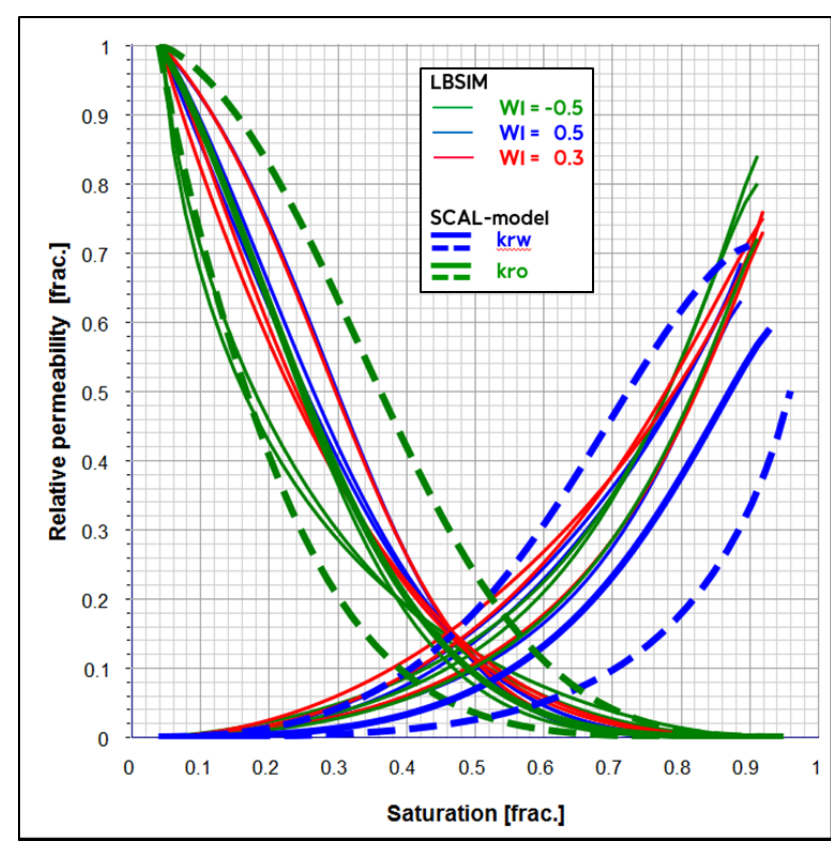

Fig. 15 Wettability sensitivity in relative permeability for Sample A. The data are compared to the SCAL model with uncertainty span (bold lines).

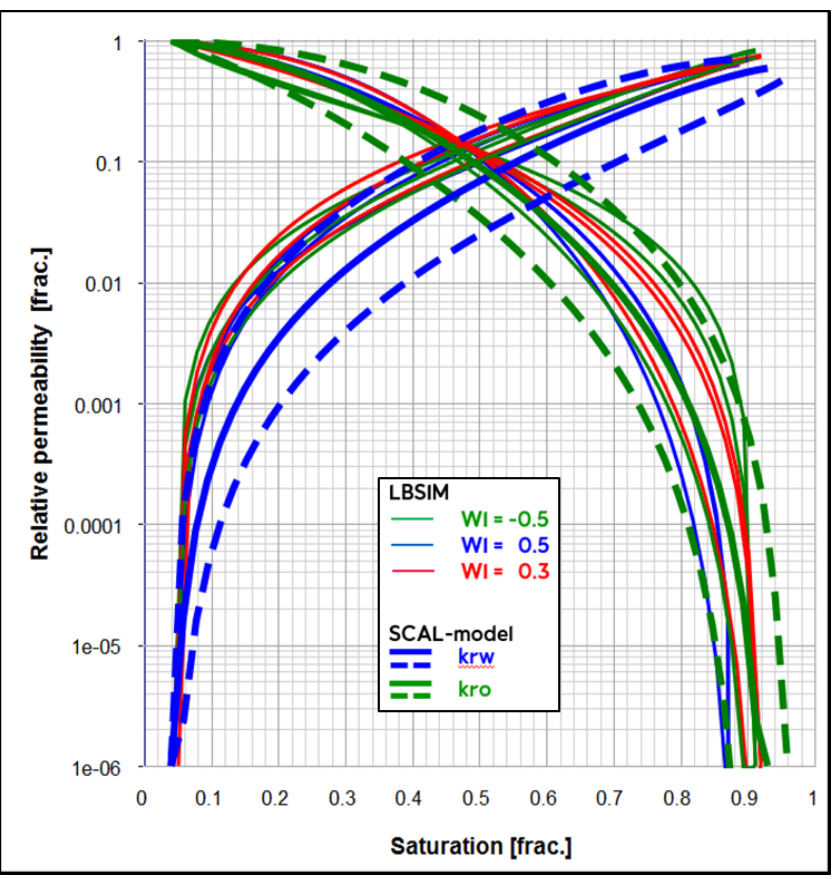

Fig. 16 Wettability sensitivity in relative permeability for Sample A. The data are compared to the SCAL model with uncertainty span (bold lines).

In terms of analysis of uncertainty on relative permeability measurements that owe to wettability, we have plotted the results for each sample in Fig. 16 to Fig. 20. The results are differentiated on sample with

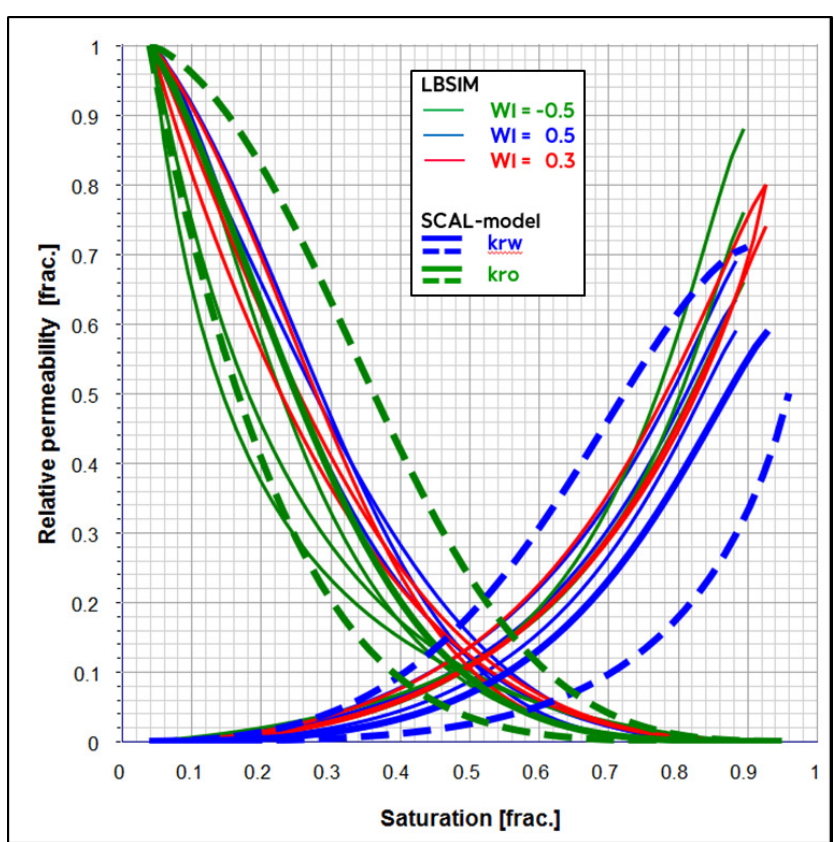

Fig. 17 Wettability sensitivity in relative permeability for Sample B. The data are compared to the SCAL model with uncertainty span (bold lines).

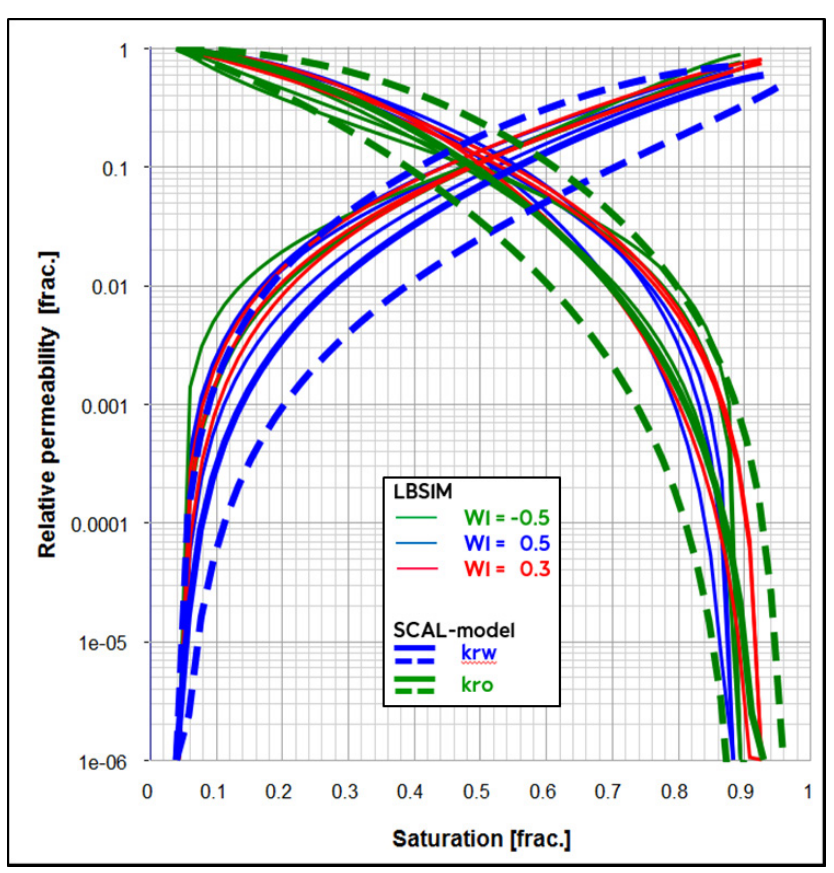

Fig. 18 Wettability sensitivity in relative permeability for Sample B. The data are compared to the SCAL model with uncertainty span (bold lines).

three different non-uniform wetting conditions. The samples are homogeneous and isotropic. Hence, the 


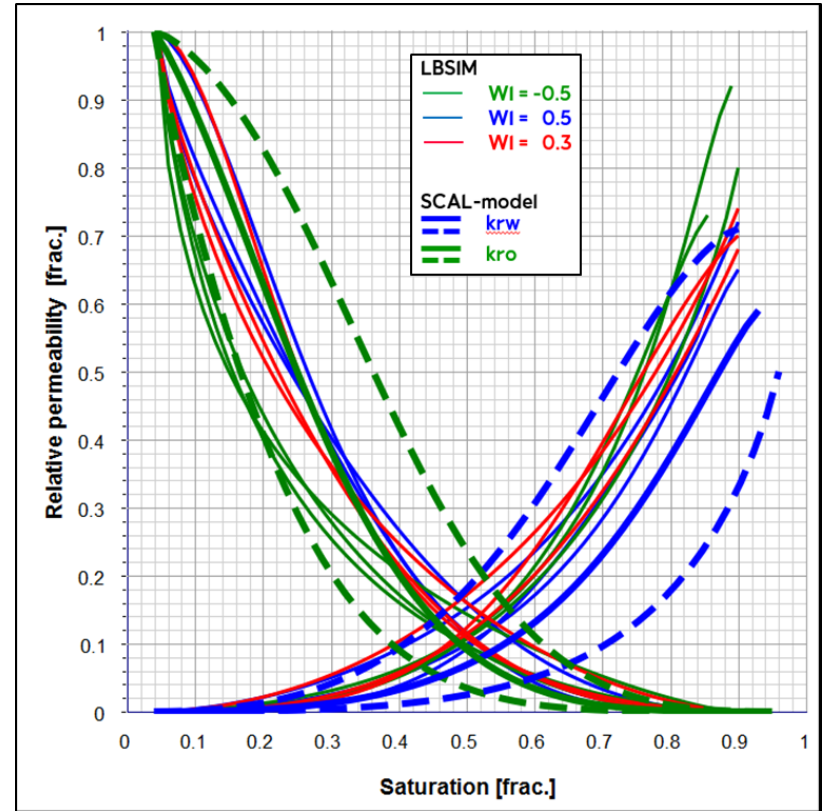

Fig. 19 Wettability sensitivity in relative permeability for Sample C. The data are compared to the SCAL model with uncertainty span (bold lines).

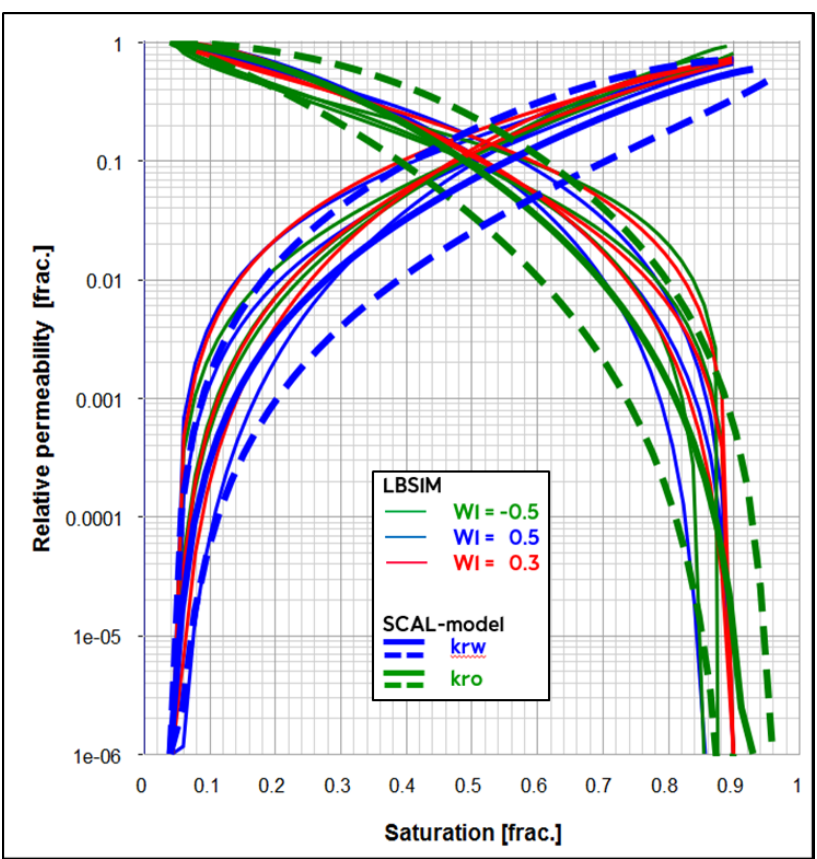

Fig. 20 Wettability sensitivity in relative permeability for Sample C. The data are compared to the SCAL model with uncertainty span (bold lines).

results from orientation should not be systematically different although we expect a certain degree of spreading in the results. Also, the SCAL models with uncertainty span should apply to all samples.

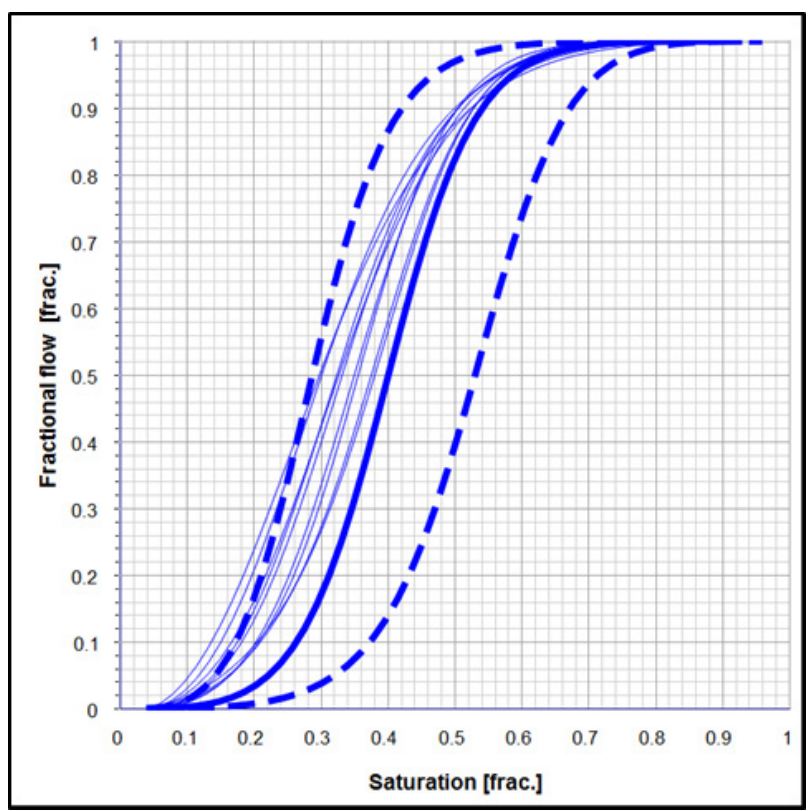

Fig. 21 Fractional flow curves for all non-uniform wettability cases Sample A.

However, we expect to see a certain trend in relative permeability owing to different wettability. With wettability towards more oil wet, the curves tend to be more pessimistic. As the wettability tends more towards the neutral wet regime, the curves are more in agreement with the SCAL model also for the end-points.

Fraction flow curves for all samples are shown in Fig. 21 to Fig. 23. Most curves fall within the uncertainty span of the SCAL model, but towards more mobile water. 


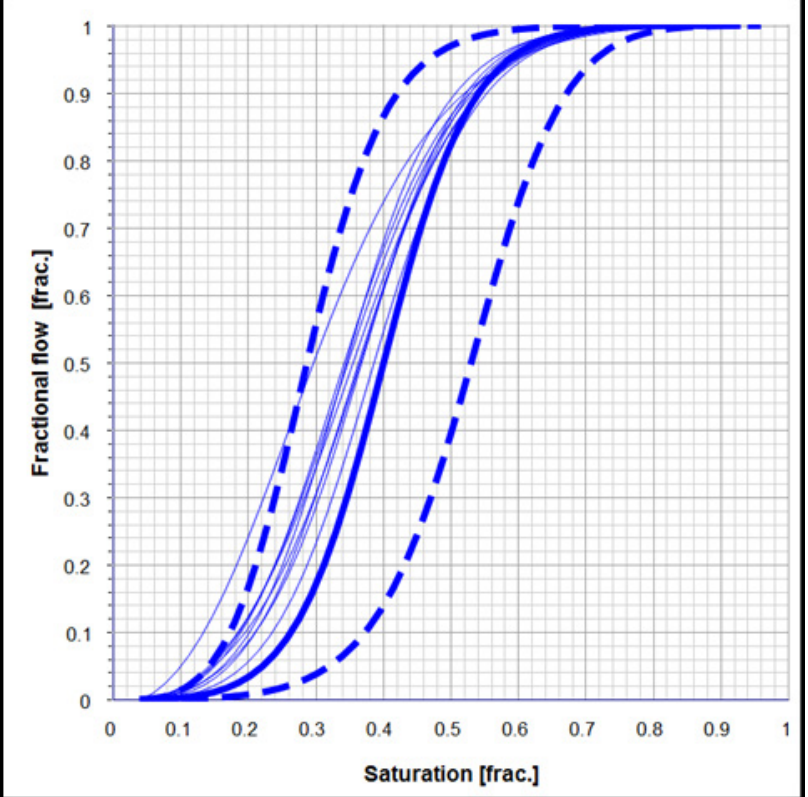

Fig. 22 Fractional flow curves for all non-uniform wettability cases Sample B.

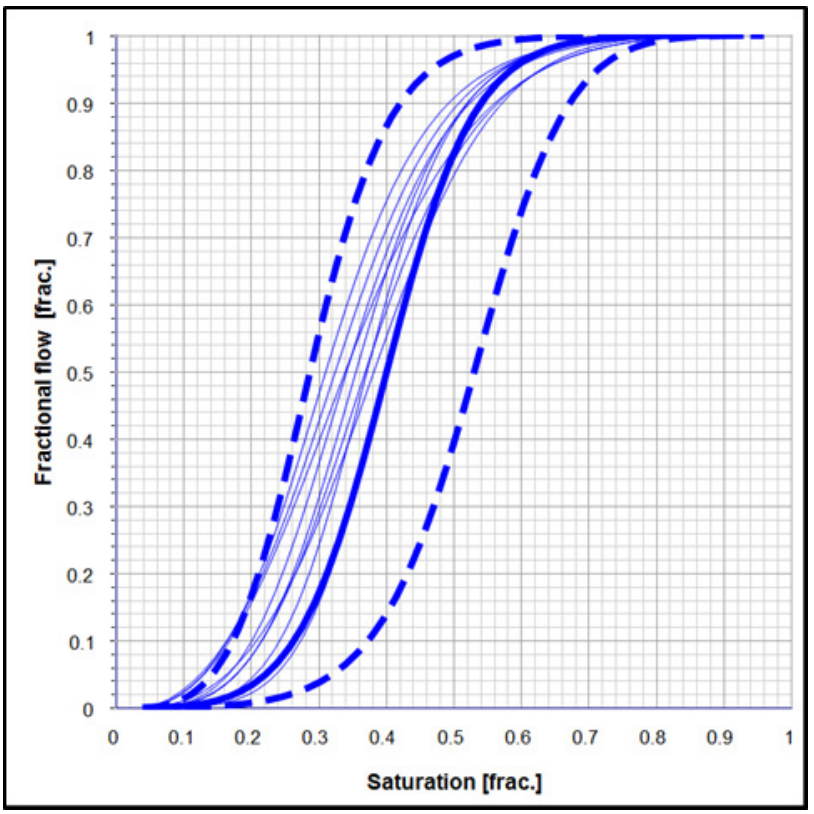

Fig. 23 Fractional flow curves for all non-uniform wettability cases Sample C.

\section{Discussion}

Pore scale simulations of the individual sample maps the steady state experiment reasonably. It also provides an estimate of the uncertainty span due to spatial- and wettability variations as shown in Fig. 11 and Fig. 12. Even though we have compared with the steady state experiment, it is not - a priori - expected that the results should map each other perfectly. The core samples used for the composite core in the steady state experiments have undergone the common "recommended practice" preparation prior to experimentation - restored state, $S_{\text {wir }}$ establishment and aging with live oil. There is no guarantee that this procedure provides the "correct" wettability-state of the core sample prior and during the flow experiment.

The relative permeability to oil and water are slightly over-estimated toward residual oil saturation, $\mathrm{S}_{\text {orw }}$, interpreting the water relative permeability, $\mathrm{k}_{\mathrm{rw}}$, slightly too pessimistic and the oil relative permeability, $\mathrm{k}_{\mathrm{ro}}$, slightly too optimistic compared to the steady state experiment. However, this is within the expected uncertainty for SCAL-experiments and multi-phase flow behaviour in general.

It needs to be emphasized, that the digital models are restrained by the resolution of the micro-CT images. Low relative permeability values close to the saturation endpoints and residuals tend to be over-estimated. It is a general short-coming of direct simulation techniques to handle very low fractional flows. There will be a certain amount of finite size effects linked to fluid-fluid interfaces and fluid-solid interactions. In general, these will lead to noise and small-scale currents that contribute relatively stronger to small fractional flow rates. Nevertheless, steady state set-ups are in general not the best candidate for end-point estimations and a combination with additional centrifuge experiments/set-up is advised.

With a detailed mapping of local wettability, we can reproduce expected trends, but also categorize properties on the pore space that are considered "Black Box" in ordinary core flooding experiments. More sophisticated wettability maps should preferably be considered for further work. A wettability map that distribute various insitu contact angels throughout a mixed-wet pore structure with local varieties depending upon pore-shapes [19]. Further the traditional classifications of wettability intermediate-wet, neutral-wet, weakly water-wet, weakly to strongly water-wet etc. - can be distributed by defining in-situ contact angels and interfacial tensions to further study variations of realistic wettabilities where none of the fluids have strong wetting preferences.

\section{Conclusion}

In this paper the following conclusions are drawn:

- $\quad$ LBSIM combined with wettability mapping and spatial directions provides reliable uncertainty span for the relative permeability curves.

- LBSIM and wettability mapping has the potential to provide basic understanding of mechanisms that influence the flow behaviour. 
- Non-uniform wettability defined from irreducible oil distribution leaves corners and small pores water wet. Such wettability distribution lowered the water relative permeability curves compared to a uniform wettability distribution. A non-uniform wettability distribution is considered a more realistic scheme.

- Uniform wettability with a weak overall wetting preference produces almost symmetric oil and water relative permeability curves and thus a more pessimistic case compared to SCAL models.

- For a fruitful and possible concluding approach, we need to properly define contact angle and interfacial tension for wettabilities.

\section{References}

1. Morrow, N.R. (editor): "Interfacial phenomena in petroleum recovery”, 1991, Marcel Dekker Inc., New York.

2. Radke, C.J., Kovscek, A.R. and Wong, H.: "A PoreLevel Scenario for the Development of Mixed Wettability on Oil Reservoirs", SPE 24880, presented at the 67th Annual Technical Conference and Exhibition of the Society of Petroleum Engineers, Washington. DC, October 4-7, 1992.

3. Anderson, W.G.: "Wettability Literature Survey Part 1; Rock/Oil/Brine Interactions, and the Effects of Core Handling on Wettability" JPT (Oct. 1986), 1125-44.

4. Anderson, W.G.: "Wettability Literature Survey Part 2; Wettability Measurements" JPT (Nov. 1986), 1246-62.

5. Anderson, W.G.: "Wettability Literature Survey Part 3; The Effects of Wettability on the Electric Properties in Porous Media” JPT (Dec. 1986), 137178.

6. Anderson, W.G.: "Wettability Literature Survey Part 4; The Effects of Wettability on Capillary Pressure" JPT (Oct. 1987), 1283-1300.

7. Anderson, W.G.: "Wettability Literature Survey Part 5; The Effects of Wettability on Relative permeability" JPT (Nov. 1987), 1453-68.

8. Anderson, W.G.: "Wettability Literature Survey Part 6; The Effects of Wettability on Waterflooding" JPT (Dec. 1987), 1605-22.

9. Amott, E.: "Observations Relating to the Wettability of Porous Rock", Trans., AIME (1959) 216, 156-62.

10. Boneau DF and Clampitt RL: "A Surfactant System for the Oil-Wet Sandstone of the North Burbank Unit" Journal of Petroleum Technology 29, no. 5 (May 1977): 501-506.
11. Donaldson EC, Thomas RD and Lorenz PB: "Wettability Determination and Its Effect on Recovery Efficiency" SPE Journal 9 (March 1969): 13-20.

12. Sendra User guide 2018.5, ProRes AS, 2019.

13. Sylte, A., Ebeltoft, E. and Petersen, E.B.: "Simultaneous Determination of Relative Permeability and Capillary Pressure Using Data from Several Experiments". Paper SCA 2004-08 presented at the International Symposium of the Society of Core Analysts, Abu Dhabi, UAE, 5-9 October 2004.

14. Lomeland, F., and Ebeltoft, E.: "A New Versatile Capillary Pressure Correlation”. Paper SCA 2008-08 presented at the International Symposium of the Society of Core Analysts, Abu Dhabi, UAE, 29 October - 2 November 2008.

15. Lomeland, F., Ebeltoft, E., and Thomas, W.H.: "A New Versatile Relative Permeability Correlation". Paper SCA 2005-32 presented at the International Symposium of the Society of Core Analysts, Toronto, Canada, 21-25 August 2005.

16. T. Ramstad, I. Nasiru, C. Nardi, P. E. Øren, "Relative permeability calculations from two-phase flow simulations directly on digital images of porous rocks", Transport in Porous Media 94, 487 - 504 (2012)

17. Lopez, O., Mock, A., Skretting, J., Pedersen EB., Øren, P., Rustad, AB., "Investigation into the Reliability of Predictive Pore-Scale Modeling for Siliciclastic Reservoir Rocks", Proceedings of the 2010 International Symposium of Core Analysts, SCA2010-23 (2010)

18. Ruspini, LC., Farokhpoor, R., Øren, P., "Pore-scale modeling of capillary trapping in water-wet porous media: A new cooperative pore-body filling model". Adv. Water Resources, (2017), 108, 1-14.

19. Lin, Q, Bijeljic, B., Krevor, S.C., Blunt, M. J., Berg, S., Coorn, A., van der Linde, H., Georgiadis, A., Wilson, O.B.: "A New Waterflood Initialization Protocol for Pore-Scale Multiphase Flow Experiments". Paper SCA 2018-32 presented at the International Symposium of the Society of Core Analysts, Trondheim, Norway, 27-30 August 2018.

20. Ebeltoft, E., Lomeland, F., Brautast, A., Haugen, Å.: "Parameter Based SCAL - Analysing Relative Permeability for Full Field Application", reviewed Proceedings of the 2014 International Symposium of the SCA, Avignon, France, September 8-11, 2014. 